\title{
Characterizing morphology in organic systems with resonant soft X-ray scattering
}

Joshua H Carpenter, Adrian Hunt, Harald Ade

Department of Physics and Organic and Carbon Electronics Laboratory (ORaCEL), NCSU, Raleigh, NC 27695-8202, United States

Abstract: Resonant soft X-ray scattering (R-SoXS) has proven to be a highly useful technique for studying the morphology of soft matter thin films due to the large intrinsic contrast between organic materials and the anisotropic nature of the resonant electronic state transitions from which the contrast originates. This allows R-SoXS users to measure spatial composition correlations from crystalline and amorphous phases in heterogeneous organic samples, infer relative domain purity, and determine average local molecular ordering correlations. R-SoXS has been used to study the morphology of organic photovoltaics, organic thin film transistors, biological systems, and block copolymer engineering applications. The mesoscopic morphological information compliments molecular packing determined with hard X-rays, so that complex structure-property relationships can be elucidated over a large range of length scales. Extensions of R-SoXS have also emerged that make use of more advanced sample setups or different experimental geometries than normal transmission, such as $\theta-2 \theta$ reflectivity or grazing incidence.

Keywords: scattering, soft X-ray, resonant scattering, morphology characterization, organic thin films, organic electronics, soft matter, block copolymers

\section{Introduction}

In the past few decades, organic materials have been synthesized that have properties traditionally associated with inorganics such as high tensile strength, and electrical and thermal conductivity [1-4]. This has enabled the development of new technologies and reengineering of existing technologies using organic materials. Often, properties of organic materials are not described well by long-standing theories developed to explain phenomena in inorganic materials, or their use in applications requires the creation of nano-structured multicomponent systems. This, coupled with the advent of nanotechnology in general, has necessitated the need to understand structure-property relationships in organic systems from the molecular to 
macroscopic length scale. Unfortunately, many of the structural probes that are used to characterize inorganic materials are often not suitable for organic systems, due to induced radiation damage, lack of contrast, spatial resolution, chemical specificity, or some combination thereof. Soft X-ray methods, in particular resonant soft X-ray scattering (R-SoXS) [5-7], have overcome many of these issues while also allowing the user to obtain a variety of information currently inaccessible through any other single technique.

In this perspective, we will first give a brief history of the evolution of R-SoXS from related techniques followed by an overview of the theoretical background that discusses the morphological information that can be gained through use of the technique. We will subsequently discuss current applications of R-SoXS, highlighting particularly salient examples, before finally discussing emerging implementations of R-SoXS and potential directions for future research. An emphasis is placed on the use of R-SoXS and aspects of analysis methodology insufficiently discussed or wrongly applied in the literature, rather than a detailed comparison to complementary methods or complete retracing of its development and historical context.

\section{Abridged R-SoXS History}

The development and power of R-SoXS as a convenient and unique characterization method can be traced to a number of confluences, but primarily rests on the advantages of near edge X-ray absorption fine structure (NEXAFS) spectroscopy [8]. NEXAFS is a specialized absorption spectroscopy near the binding energy of core shell electrons in low-Z atoms. Intense, sharp peaks near the absorption edge are characteristic of resonant electronic transitions from an atomic core level to unoccupied anti-bonding states. The energy and intensity of these resonant absorption features are highly dependent on the type, orientation, and number of bonds an atom forms in a specific molecule as well as the participation of hetero-atoms in the bonding. Therefore NEXAFS spectra are especially useful for differentiating organic materials, which are very similar in terms of their constituent atoms but different in terms of the local bonding environment seen by each atom. A number of spectral databases can be consulted that have documented this spectral richness [9-11]. Applications of NEXAFS and resonant scattering are also prevalent for a wide range of magnetic transition metal compounds [12, 13]. The intrinsic chemical differentiation of infrared and nuclear magnetic resonance spectroscopy is higher than that of NEXAFS [14], but these methods cannot be made surface sensitive or be incorporated into high spatial resolution microscopy or scattering methods.

Morphological characterization techniques that employ X-ray absorption can exploit the differences between various materials measured by NEXAFS. One such technique, scanning transmission X-ray microscopy (STXM) [15], can be used to map each component in a sample by imaging at different resonant energies and extracting the composition via singular value decomposition procedures [16]. Compositional mapping in carbon materials was first 
demonstrated in 1992 [17] using resonant energies near the carbon K-edge, followed in 1993 with applications to map molecular orientation [18]. Surfaces can also be mapped in STXM with the detection of secondary electrons $[19,20]$ or in photoemission microscopes [21]. STXM has since become a vital tool for characterizing organic thin film morphology [16, 22].

Modern STXM instruments have a spatial resolution of $\sim 30 \mathrm{~nm}$ [23], corresponding to the diffraction limit of the optical elements used. This is slightly too large to study many important organic systems, further complicated by the suppressed contrast over a large spatial frequency range that complicates quantitative analysis [24]. Also, because mapping different components takes advantage of absorption and not phase contrast, there is a significant risk of radiation damage to the sample [25-27]. Furthermore, STXM is not typically used in tomography mode $[28,29]$ and 3-Dimensional features in the 50-300 nm thick films investigated are projected onto 2-Dimensional maps, often leading to overlapping information and reduced apparent contrast. Though a powerful technique in its own right and still used extensively by many researchers [30], these current limitations of STXM have necessitated the development of $\mathrm{R}$-SoXS [5-7] as well as ptychography methods reaching $\sim 5 \mathrm{~nm}$ resolution [31-33]. R-SoXS can extract statistical information of spatial frequency distributions at corresponding length scales below the spatial resolution of STXM while also acquiring data with significantly reduced radiation dose and thus risk of damage to the sample compared to STXM. Furthermore, scattering does not suffer from the 2-Dimensional projection of 3-Dimensional information in thicker films (see below). For a more complete description of STXM technology and its applications in a range of fields, see the paper by Hitchcock et al. in this issue [34].

$\mathrm{R}-\mathrm{SoXS}$ data is recorded as scattered intensity at a given point in reciprocal space. The phase information of the scattered radiation is lost. Real space information extracted from 2Dimensional scattering data corresponds to the average physical structure over the entire illuminated area, with a spatial resolution limit of $\lambda / 2$, in complete backscattering geometry, where $\lambda \approx 4.5 \mathrm{~nm}$ at the carbon K-edge. In comparison to STXM, spatial resolution is gained at the expense of location information. Since the radiation is spread over a much larger sample area, R-SoXS can differentiate between multiple components in a sample with significantly less radiation damage than STXM. Furthermore, R-SoXS is highly phase sensitive so data can be acquired with good contrast below an absorption edge [35]. We also note that the underlying physics of resonant scattering methods have been exploited previously in the hard X-ray regime, where they are referred to as anomalous X-ray scattering [36-40].

\section{R-SoXS Theoretical Background and Analysis Methodology}

\subsection{Optical Constants and Contrast}

A complete theoretical treatment of R-SoXS is beyond the scope of this perspective, however a brief overview serves to help highlight what makes it such a powerful tool. At soft Xray energies, the complex index of refraction of a material is conventionally written as $\tilde{\mathrm{n}}(\mathrm{E})=$ 
$1-\delta(E)+i \beta(E)$, where $E$ is energy. Note that we have explicitly denoted the energy dependence of the optical constants. $\beta(E)$ is proportional to the absorption coefficient from NEXAFS spectra and $\delta(E)$ can be calculated from $\beta(E)$ via the Kramers-Kronig relations [11, 41-44]. Thus $\tilde{\mathrm{n}}(\mathrm{E})$ near an absorption edge inherits a strong, molecule-specific energy dependence from the resonant peaks observed in the absorption spectrum so differences in $\tilde{\mathrm{n}}(\mathrm{E})$ can also be a means for distinguishing between materials. Given that the long wavelength of soft $\mathrm{x}$-rays result in sampling many atoms of a sample, we prefer the language of optics and its emphasis on bulk properties, rather than the use of scattering factors prominent in the field of hard x-ray scattering. Since resonant electronic transitions near an absorption edge are often highly anisotropic for polarized radiation, we note that, in general, the complex index of refraction should be a second rank tensor $\widetilde{\mathbf{n}}(\mathrm{E})$. We will return to this point later, but for now we will assume that $\tilde{\mathrm{n}}(\mathrm{E})$ is scalar.
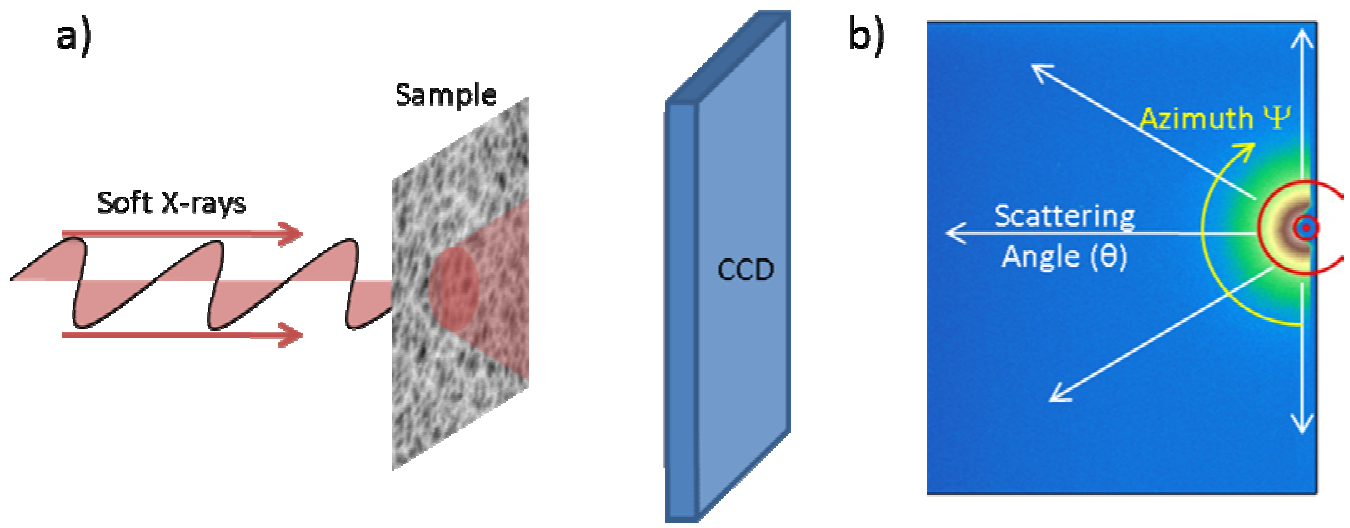

Fig. 1. A schematic of a standard transmission R-SoXS experiment is shown in a). 2-Dimensional scattering data is collected in transmission from a soft X-ray beam incident normally on the sample, an example of which is shown in b). The white arrows indicate the scattering angle $\theta$ and the yellow arrow indicates the azimuthal angle, which is averaged over to obtain a 1-Dimensional I(q) profile. Figure Reprinted by permission from Macmillan Publishers Ltd: Nature Materials [45] with permission, copyright 2012.

The R-SoXS intensity in reciprocal space is determined by the real space morphology of a sample. Standard R-SoXS employs a small angle scattering transmission geometry, with a thin film perpendicular to an incoming soft X-ray beam, as shown in Fig. 1 a). The scattered intensity is recorded by a 2-Dimensional detector behind the sample, and the position on the detector is converted into the scattering vector ${ }^{1} \mathbf{q}=\mathbf{k}_{0}-\mathbf{k}$, where $\mathbf{k}_{0}$ and $\mathbf{k}$ are the incident and scattered wave vectors, respectively, and $|\mathbf{q}|=4 \pi \sin (\theta) / \lambda$ where $\theta$ is defined relative to $\mathbf{k}_{0}$. Noting that soft $\mathrm{X}$-ray wavelengths are much greater than the Bohr radius, $1-\delta$ and $\beta$ are proportional to the real and imaginary parts, respectively, of the complex scattering factor [42, 43]. Therefore the scattered intensity $\operatorname{I}(\mathbf{q}) \propto|\Delta \tilde{n}(\mathbf{q})|^{2}$ where $\Delta \tilde{n}(\mathbf{q})$ is the Fourier transform of $\Delta \tilde{\mathrm{n}}(\mathbf{r})$, the difference in $\tilde{\mathrm{n}}$ between two points in the sample physically separated by $\mathbf{r}$. In other

\footnotetext{
${ }^{1}$ As R-SoXS literature to date has used $\mathbf{q}$ as a reciprocal space coordinate, we will use this convention as well, but the spatial frequency $\mathbf{s}=\mathbf{q} / 2 \pi$ may be more intuitive when interpreting real space morphological information.
} 
words, the Fourier transform of the composition variations of the sample at a given length scale determine the scattered intensity.

In the simplest model system, a film of a pure material, $\Delta \tilde{\mathbf{n}}(\mathbf{r})$ is only nonzero between sample and vacuum or due to density fluctuations in the sample. If the sample consists of multiple materials, $\mathrm{I}(\mathbf{q})$ can be expressed as a linear combination of $\left|\Delta \tilde{\mathrm{n}}_{\mathrm{ij}}(\mathbf{q})\right|^{2}$ between materials $\mathrm{i}$ and $\mathrm{j}[46,47]$. It is therefore helpful to define the R-SoXS contrast function $\mathrm{C}_{\mathrm{ij}}(\mathrm{E})=$ $\mathrm{E}^{4}\left|\Delta \tilde{\mathrm{n}}_{\mathrm{ij}}(\mathrm{E})\right|^{2}=\mathrm{E}^{4}\left(\Delta \delta_{\mathrm{ij}}(\mathrm{E})^{2}+\Delta \beta_{\mathrm{ij}}(\mathrm{E})^{2}\right)$ using the intrinsic $\tilde{\mathrm{n}}(\mathrm{E})$ of pure materials to determine the relative contribution to the scattered intensity from a specific pair of materials at a given energy. $\Delta \tilde{\mathrm{n}}_{\mathrm{ij}}$ can be substantial near an absorption edge so R-SoXS contrast between two organic materials is often orders of magnitude higher than those of other techniques used to characterize the morphology of organic systems, such as small angle X-ray scattering with hard X-rays (SAXS) or transmission electron microscopy (TEM), which utilize electron density difference as a contrast mechanism. Fig. 2 a) and d) illustrate the difference in contrast from soft to hard $\mathrm{X}$ rays for two materials. With appropriate choice of X-ray energy, R-SoXS can probe the same morphological structures as these other techniques or can probe other structures not detectable from differences in electron density. If the R-SoXS energy is chosen so that the contrasts of two materials relative to some third phase are identical, they are contrast-matched [48, 49]. By probing a sample with a series of energies that contrast-match each pair of materials, their contributions to the scattered intensity can be determined. This capability to control the scattering contrast between different materials without sample modifications is one of the biggest strengths of R-SoXS. 


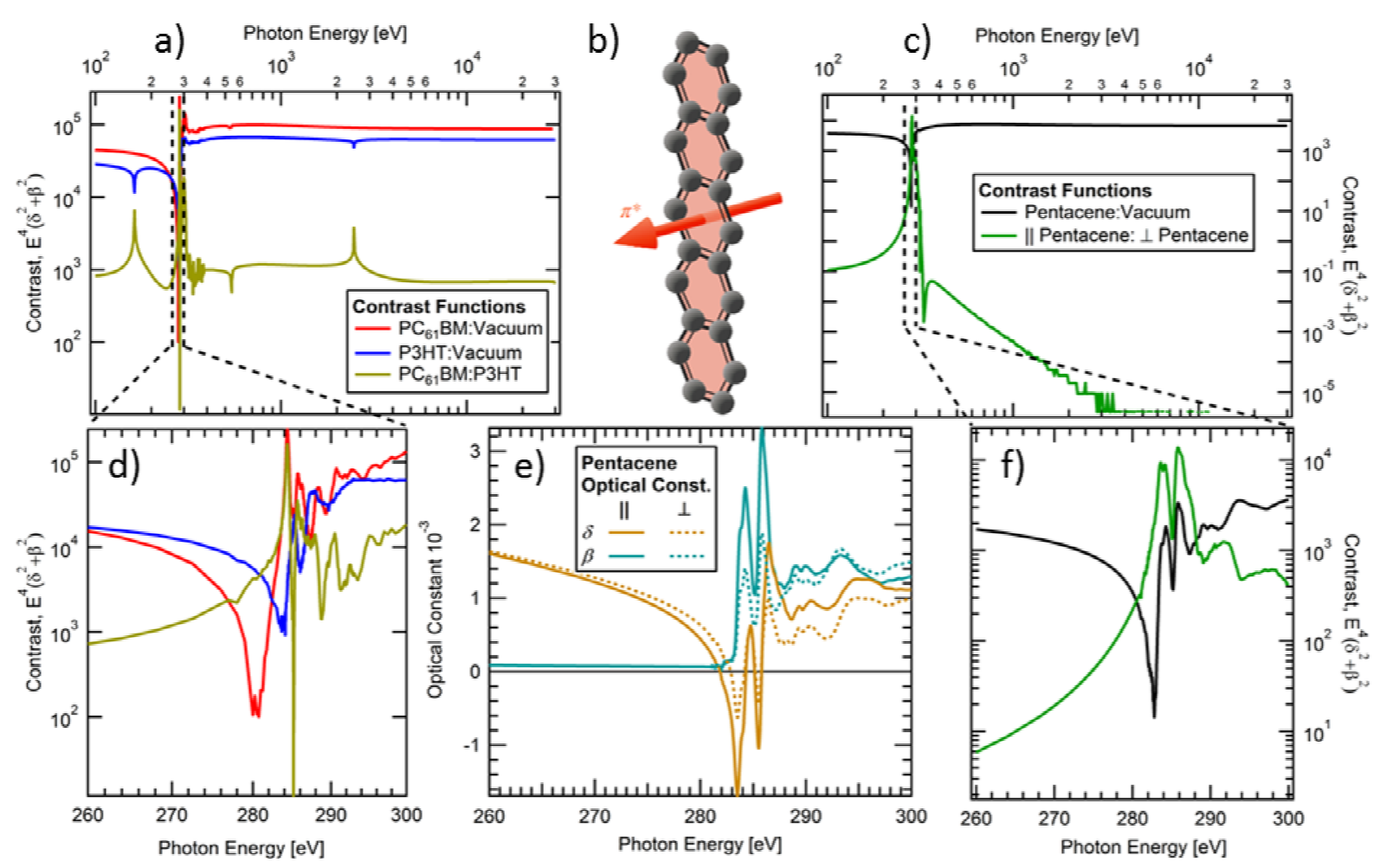

Fig. 2. a) Relevant vacuum and material contrast functions plotted over a large photon energy range for a common organic photovoltaic system (P3HT:PCBM). d) is an expanded view of a) for the energy range about the carbon Kedge $(\sim 284 \mathrm{eV})$. An illustration of the $\mathrm{C}-1 \mathrm{~s} \rightarrow \pi_{\mathrm{C}=\mathrm{C}}^{*} \mathrm{TDM}$ for pentacene is shown in $\left.\mathrm{b}\right)$. The optical constants of pentacene with this TDM perpendicular or parallel to polarization of the incoming electric field are shown in e). Vacuum and orientation contrast, calculated from the optical constants of pentacene (a shortened range of which are shown in e), are plotted over a large photon energy range in c). f) is an expanded view of c) for an energy range about the carbon K-edge. The figure has been adapted and reprinted by permission from Macmillan Publishers Ltd: Nature Materials [45], copyright 2012.

R-SoXS can also provide information on relative local molecular orientation in a sample [45, 50-52]. Most often, samples studied by R-SoXS are isotropic overall (i.e. "globally") but molecular orientation can be highly correlated locally, such as in systems with preferential orientation at interfaces between different phases or fibrillar aggregates [45, 50]. As noted earlier, the cross section of a resonant carbon K-edge transition is dependent upon bond orientation relative to the polarization of the incoming radiation. This means that the optical constants near an absorption edge are, generally speaking, second rank tensors. The dependence of the optical constants upon molecular orientation with respect to the polarization of the incident beam can, in principle, be calculated by fitting angle-resolved NEXAFS spectra from highly ordered samples $[8,53]$. Often this calculation is not necessary, because symmetries of the molecule can be used to simplify the optical constant tensors. For example, in conjugated organic materials, the $\mathrm{C}-1 \mathrm{~s} \rightarrow \pi_{\mathrm{C}=\mathrm{C}}^{*}$ transition is often the dominant absorption feature observed in NEXAFS spectra. The transition dipole moment (TDM) of this excitation has uniaxial symmetry perpendicular to the conjugated plane, as illustrated for pentacene in Fig. 2 b), that 
allows the elements of the optical constant tensors of an arbitrarily oriented molecule to be expressed well by a linear combination of their values when the incident radiation is parallel or perpendicular to this TDM $[45,47]$. This is especially true at the energy that corresponds to the TDM in question, which is, in practice, the energy that is used to probe the sample.

In a polarized R-SoXS (P-SoXS) experiment, each material in a sample can be considered two "materials" - one for each of the two orthogonal orientations of a specific TDM with respect to the polarization of the beam. In the same way that material contrast functions are calculated for R-SoXS, P-SoXS orientation contrast functions are calculated from their optical constants and energies are chosen with which to probe the samples based on these functions. The optical constants for the respective orthogonal orientations of the $\mathrm{C}-1 \mathrm{~s} \rightarrow \pi_{\mathrm{C}=\mathrm{C}}^{*} \mathrm{TDM}$ in pentacene are shown in Fig. 2 e). From these orientational optical constants, the orientation contrast function is calculated and plotted over a large photon energy range in Fig. $2 \mathrm{c}$ ), with an expanded view of the energy range about the carbon K-edge in Fig. 2 f). The polarization dependence of scattering has also been exploited in reflectivity and off-specular geometries and used to help characterize depth-dependent molecular ordering in organic thin films [54-56], as well determine orientation in liquid crystalline systems [57].

\subsection{Extracting Morphological Information from R-SoXS Data}

Once the contrast functions which are relevant to a given sample are known and a suitable energy is chosen to investigate the structures of interest, the detector records a 2Dimensional map of a section of the 3-Dimensional I(q) pattern. Most standard R-SoXS experiments utilize relatively small angles $\left(<30^{\circ}\right)$ and so the component of $\mathbf{q}$ out of the plane of the sample is generally negligible and the measured real space composition fluctuations are interpreted to be in the plane perpendicular to the normal of the sample. Any conventional quantitative analysis first reduces the 2-Dimensional data to an average 1-Dimensional I(q) profile by averaging over an appropriate ${ }^{2}$ azimuthal range about the beam center.

The interpretation of $\mathrm{I}(\mathrm{q})$ and any further analysis must be done carefully, while considering both the experimental geometry and the structural symmetries in the sample. The simplest model independent analysis method is to directly convert peak and shoulder positions to real space characteristic lengths in the sample, also known as the long period [58]. The energy and polarization dependence of the peaks provide information about the structure in the sample from which the scattering originated [59, 60]. Domain spacings in real-space calculated in this manner have been shown to correlate with those measured by other techniques [61], but the data can be further processed to better represent the true spatial frequency distribution in the sample. More complex analysis can yield the cord length distribution and various correlation functions. However, these typically already assume a binary sample morphology and are thus not truly model independent. Quite often, small angle scattering is analyzed by forward simulations in

\footnotetext{
${ }^{2}$ 2-Dimensional data that is observed to be isotropic is averaged over the full azimuthal range. Anisotropic scattering data and the azimuthal averaging used to analyze them will be discussed later in this section.
} 
which a morphology is modeled and its scattering is compared to the data. Variations of the morphology are then evaluated until a best fit is found.

If the sample is assumed to be globally isotropic then the scattering pattern should not change upon any arbitrary rotation of the sample. Thus, to give an accurate representation of the spatial frequency distribution in the sample, I(q) needs to be corrected for the fraction of the reciprocal space surface at equal q being sampled. This is known as Lorentz correction [58, 62]. Under the assumption of a globally isotropic 3-Dimensional morphology ${ }^{3}$, the multiplicative Lorentz correction factor is proportional to $q^{2}$ [58]. It has been shown for SAXS data [58, 63], as well as simulated R-SoXS data [46], that the long period extracted from Lorentz corrected profiles gives the best agreement to real-space domain spacings present in a sample, as compared to similarly complex analysis methods. This has been shown to be true for R-SoXS data with multiple characteristic length scales as well $[46,59]$. However, a peak in Lorentz corrected data should not blindly be interpreted as a long period. A sample of isolated low density spheres would also produce a peak in Lorentz corrected data, yet there is no defined spacing in such a sample. The respective phases in a sample need to have high and comparable volume fractions to yield a peak that corresponds to a spacing. We also want to emphasize that in cases where it is known that there is strong asymmetry in the sample, as in the out-of-plane direction with respect to the sample, Lorentz correction is not strictly appropriate. For example, features at small $\mathrm{q}$ corresponding to purely lateral structures larger than the sample thickness will obviously not exhibit 3-Dimensional symmetry and the intensity of such features in a Lorentz corrected plot would be too high. Direct and model independent analysis of Lorentz corrected data is often though a very convenient and powerful method.

The Lorentz corrected spatial frequency distributions acquired with R-SoXS of OPV morphologies are often log-normal [35, 59, 60]. Log-normal probability density functions frequently occur in nature when a variable is the multiplicative product of many independent random variables. We note that log-normal distributions in Lorentz corrected data also have a peak in uncorrected data, and thus do correspond to morphologies that exhibit well defined spacing (see Fig. S1, supplemental). Converting a spatial frequency distribution into a real-space distribution is possible by variable transformation. For an example, see supplemental information. There is at least one instance in R-SoXS literature where such lognormal distributions in $\mathrm{q}$ space were converted directly to real space domain size distributions without accounting for the chain rule [35], consequently underestimating the prevalence of small domains.

One way R-SoXS data could be further analyzed is with various distribution functions, such as an interface distribution function (IDF), or a chord length distribution (CLD) function [46, 58, 64-67]. We would like to highlight such functions because they are alternative representations of real space structure in the sample, though they have not been frequently utilized in R-SoXS analysis to date. The CLD measures the distance traveled along a randomly

\footnotetext{
${ }^{3}$ A sample whose structure exhibits only 2-Dimensional symmetry in the sample plane axis would have a Lorentz correction factor proportional to $\mathrm{s}$.
} 
oriented chord in one material before reaching an interface. In many applications, such as organic photovoltaics (OPVs), this appears to be the ideal data that R-SoXS could provide. Extensions of the CLD for higher dimensions and anisotropic materials have been demonstrated in SAXS analysis [58, 68] and could offer exciting potential directions for new R-SoXS analysis methods. However, IDF and CLD analysis assumes a two-phase morphology of fixed phase composition. This is often not the case in non-equilibrium structures such as OPV active layers in which mixed domains of variable composition can occur. In these samples, the information about volume fraction and composition variations, i.e. purities, are convolved and direct analysis of log-normal distributions is preferred.

Lorentz corrected profiles can also be used to measure relative composition variations $[35,69,70]$. As described above, $\mathrm{I}(\mathrm{q}) \propto|\Delta \tilde{\mathrm{n}}(\mathrm{q})|^{2}$, so that the integrated scattering intensity (ISI) can be given by

$$
\text { ISI }=\int_{q_{1}}^{q_{2}} I(q) q^{2} d q \propto V \varphi(1-\varphi)|\Delta \tilde{n}|^{2}
$$

where $\mathrm{V}$ is the scattering volume, and $\varphi$ is the volume fraction of one of the materials. If the ISI is corrected for $\mathrm{V}$ and $\varphi(1-\varphi)$, it is a measure of $\Delta \tilde{\mathrm{n}}^{2}$, i.e. the average compositional variations, over the spatial frequencies from $\mathrm{q}_{1}$ to $\mathrm{q}_{2}$. Differences in $\Delta \tilde{\mathrm{n}}^{2}$ between two samples composed of the same materials can be attributed to differences in relative composition variations averaged over the length scales probed. Two pure phases yield maximum ISI, completely mixed phases have ISI=0. ISI can thus be interpreted are the relative average purity of all phases. In R-SoXS literature to date, comparisons of relative composition variations have often performed this integration over all $\mathrm{q}$ by extrapolating the measurements. This is equivalent to calculating Porod's invariant [35, 62, 69-72], but the extrapolation makes unjustifiable assumptions that indeed a scaling relation will hold at high q. Yet, that scaling invariably breaks down at a length scale corresponding to the molecular level [73]. Though we note that Porod's invariant can be used to calculate other morphological parameters, use of ISI is more appropriate in this case. This is for several reasons, the first being to avoid confusion about the purpose of the integration. Second, by limiting the integration to a spatial frequency range of interest, the average composition variations over specific length scales can be discussed, similar to compositional maps in microscopy with limited field of view and spatial resolution. We caution though. Unlike real space information where purity and volume fraction of domains with a given size can be independently assessed, that is not the case in the reciprocal space data obtained from R-SoXS. A large volume fraction of impure small domains cannot be distinguished from a small volume fraction of pure domain. Both these cases might give the same scattering intensity at the corresponding spatial frequency. This is, however, not a problem for samples with a single lognormal distribution.

For P-SoXS, the details of quantitative analysis depend on the type of information desired and the specifics of the sample. For example, a pure material in a liquid crystalline phase can 
exhibit short-range local order [45]. 2-Dimensional P-SoXS data acquired at resonance that is azimuthally symmetric about the beam center can be processed and analyzed in much the same way as R-SoXS data. Domain spacings extracted from such data would be indicative of the local orientational coherence length (OCL) in the sample.
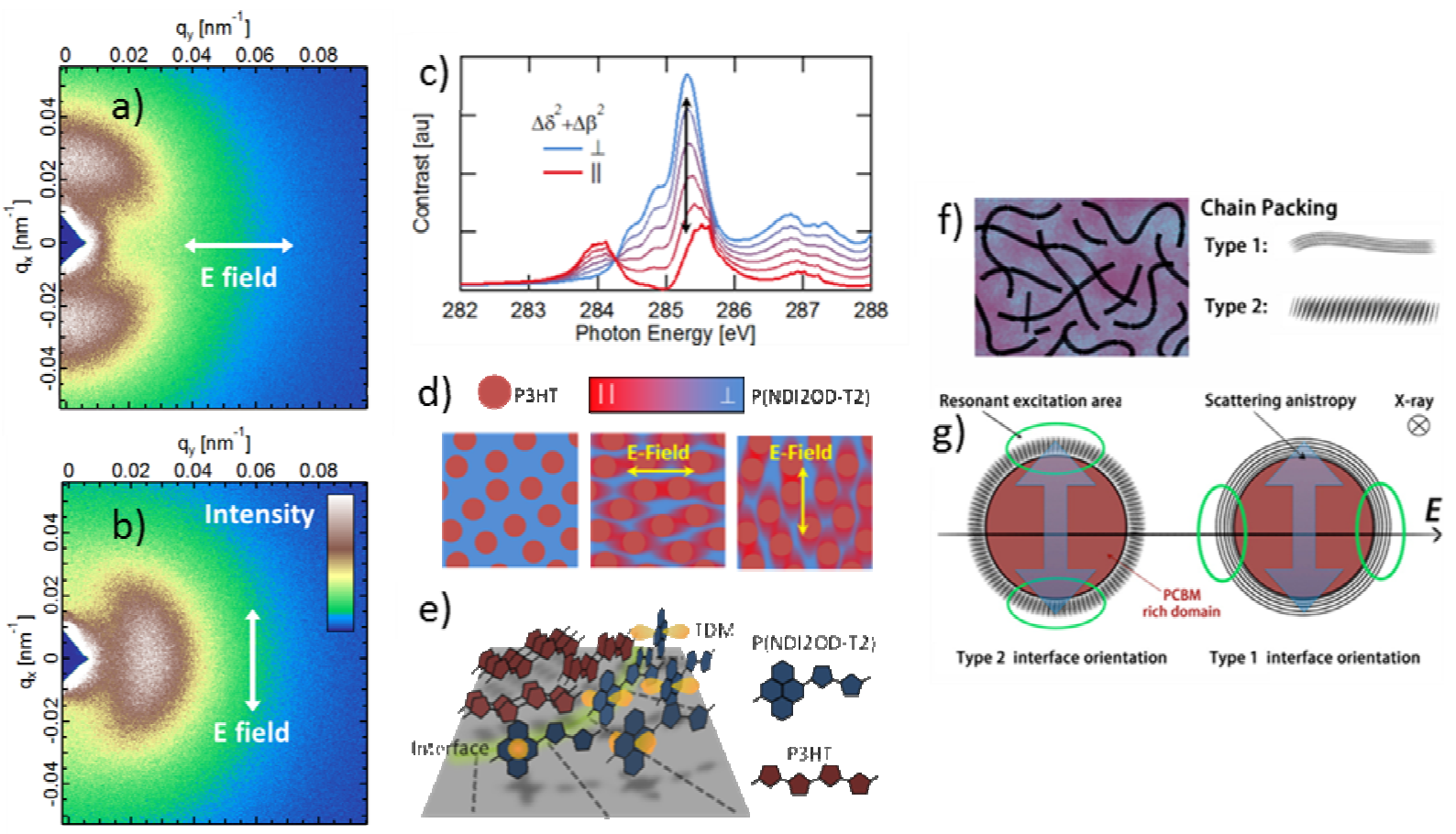

Fig. 3. Anisotropic 2-Dimensional scattering data are shown in a) and b). An illustration of preferentially oriented $\mathrm{C}-1 \mathrm{~s} \rightarrow \pi_{\mathrm{C}=\mathrm{C}}^{*} \mathrm{TDM}$ at a material interface, which can lead to such anisotropic scattering, is shown in e). In c) a contrast function for different orientations of the polymer P(NDI2OD-T2) is shown, which uses a color scale between orientations that is mirrored in d), an illustration of the scattering contrast for a given electric field of the $\mathrm{P}(\mathrm{NDI} 2 \mathrm{OD}-\mathrm{T} 2$ ) at material interfaces in the bulk. a) through e) have been Reprinted by permission from Macmillan Publishers Ltd: Nature Materials [45], copyright 2012. f) and g) have been adapted with permission from [74]. Copyright 2013 American Chemical Society.

A different analysis approach is needed for samples with anisotropic scattering data, as exemplified by Fig. 3 a) and b). In these cases, molecules might be preferentially oriented relative to interfaces between phases due to the presence of the interface, as illustrated in Fig. 3 d) and e), or fibrillar structures form during film formation and the space between fibrils is filled in by the second material as in Fig. $3 \mathrm{f}$ ) and g) [74]. The details of inferring the mechanism and actual morphology will require sophisticated modeling [46, 47]. A phenomenological morphological parameter describing such orientation/morphology correlations is the degree of molecular ordering (DMO). To quantify the DMO in a system, 2-Dimensional P-SoXS data are first azimuthally averaged over sectors either perpendicular or parallel to the polarization of the incoming beam [47, 50], Lorentz corrected and integrated over q, yielding $\mathrm{ISI}_{\perp}$ and $\mathrm{ISI}_{\|}$, 
respectively. Subsequently, the difference over the sum of the sector intensities yields the anisotropic ratio (A),

$$
\mathrm{A}=\frac{\mathrm{ISI}_{\perp}-\mathrm{ISI}_{\|}}{\mathrm{ISI}_{\perp}+\mathrm{ISI}_{\|}}
$$

The DMO can be obtained from A by calculating the extreme values of A from perpendicular and parallel reference spectra and then fitting to the experimentally measured A with a constant multiplicative factor. This multiplicative factor is the DMO. It ranges from 1 to -1 , and indicates how close the local average TDM is to being perfectly perpendicular or perfectly parallel to the incoming beam at the domain interface being probed.

As described above, R-SoXS is a valuable tool for quantifying various aspects of morphology in organic samples. It can provide equivalent domain spacing information to other techniques such as SAXS and TEM [61], while also offering molecule-specific, controllable contrast to facilitate the study of heterogeneous materials. R-SoXS can also provide information about the local molecular ordering of non-crystalline phases, which is unique among scattering techniques used to characterize the morphology of organic samples. R-SoXS shares its tunable contrast and sensitivity to molecular orientation with STXM [18, 75], but without the disadvantages and limitations. Near the carbon K-edge, the spatial frequency range of standard transmission R-SoXS can extend from greater than $0.1 \mathrm{~nm}^{-1}$ corresponding to domain spacings of less than $10 \mathrm{~nm}$, to below $0.001 \mathrm{~nm}^{-1}$, corresponding to domain spacings of greater than $2 \mu \mathrm{m}$. The upper limit of this range is higher than in a typical SAXS experiment and lower than the current spatial resolution limit in STXM. Other R-SoXS geometries can probe an even wider range of domain spacings ranging from $5 \mu \mathrm{m}$ to less than $3 \mathrm{~nm}$ [76]. Also, the same contrast principles utilized in R-SoXS has been utilized in a $\theta-2 \theta$ reflectivity geometry. Resonant soft $\mathrm{x}-$ ray reflectivity (R-SoXR) has been used to investigate a number of thin films to measure interfacial roughness with high sensitivity and specificity [54, 77-80].

\section{Applications}

\subsection{Block Copolymers}

In a 2012 study, Wong, et al. [49] utilized the contrast-matching capability of R-SoXS to verify a three-phase morphology in a block copolymer (BCP) thin film. Equivalent "indexmatching" can be achieved in neutron scattering through careful deuteration [81], whereas the contrast-matching capability of R-SoXS are intrinsic, possible for many systems, and arise from dispersion and absorption contributions, i.e. $\delta$ and $\beta$, respectively. The BCP of interest was a mesoporous polystyrene-block-polyethylene-block-polystyrene (SES) copolymer membrane fabricated by casting a blend of the copolymer and polystyrene homopolymer (PS) then using a selective solvent to remove the PS to create voids. The fabrication procedure was designed to produce a three-phase morphology consisting of a structural network of polyethylene (PE), a 
network of pores (pores/"vacuum"), and a PS liner for the pores consisting of the PS-block of the SES. The ratio of the molecular weight of the homopolymer PS to the block copolymer PS, defined as $\alpha$, was varied and R-SoXS was able to determine at what value of $\alpha$ the procedure best produced the desired morphology. We will focus here on data from the sample that had a morphology closest to that which was intended, $\alpha=0.36$.

Fig. $4 \mathrm{~b}$ ) shows the contrast functions for each of the three possible domain interfaces in this system with three energy ranges highlighted near 280, 284, and $300 \mathrm{eV}$. Cartoons depicting the contrast matching that should occur in the membrane at these energies are also shown in Fig. $4 \mathrm{~b}$ ). At $280 \mathrm{eV}$ the PS and vacuum are contrast-matched, at $284 \mathrm{eV}$ the PE and vacuum are contrast-matched, and at $300 \mathrm{eV}$ the PS and PE are contrast-matched. R-SoXS data were acquired at energies from 280 to $284 \mathrm{eV}$ and scanning electron microscopy (SEM) and SAXS were collected to aid in the interpretation of the contrast-matched R-SoXS data.


Fig. 4. 1-Dimensional scattering profiles, offset for clarity, are shown in a). A plot of three contrast functions with different contrast-matched regions highlighted and their accompanying cartoons illustrating the contrast-matching occurring are shown in b). The 1-Dimensional SAXS profile $(10 \mathrm{keV})$ of pure SES melt $\left(150^{\circ} \mathrm{C}\right)$ is shown in c). The figure has been adapted from with permission from [49]. Copyright 2012 American Chemical Society.

The SAXS and R-SoXS profiles of the BCP membrane are shown in Fig. 4 a), with their intensities offset for clarity. The SAXS profile and the $280 \mathrm{eV} \mathrm{R-SoXS} \mathrm{profile} \mathrm{are} \mathrm{strikingly}$ similar. Recall that the contrast mechanism for SAXS is electron density difference, which implies that the average pore to pore spacing in the plane of the membrane should dominate the SAXS profile due to the weak contrast between PS and PE phases. This explanation of the observed spacing is consistent with the $280 \mathrm{eV}$ R-SoXS data, where contrast between the domains is matched and "vacuum" contrast dominates the scattering. Note that the peaks are rather broad, indicating a distribution of spacings or lack of long term order, rather than a welldefined periodic structure. Quantitatively, the peak locations of this shared feature correspond to 
real spacings of $57.9 \mathrm{~nm}$ from SAXS (in the melt) and $51.2 \mathrm{~nm}$ from R-SoXS. These values were also corroborated by SEM of the membrane's surface; line scans of SEM images measured the pore to pore spacing to be $53.4 \mathrm{~nm}$.

As the R-SoXS energy is increased incrementally from 280 to $284.2 \mathrm{eV}$, a second feature becomes prominent at higher q. The source of this feature can be ascertained from Fig. $4 \mathrm{c}$ ), the SAXS profile of a pure SES copolymer acquired at $150{ }^{\circ} \mathrm{C}$ (above the melting temperature of the polymer). Though the contrast between copolymer blocks at hard X-ray energies should be very small, in the melt it should be the only contrast mechanism. Thus the observed features in Fig. 4 c) are the first and second order peaks of the characteristic spacing between copolymer blocks $(41.3 \mathrm{~nm})$. Note how the peaks of pure SES are relatively low intensity because of the weak contrast, though very narrow because of the regularity of the spacing in a pure block copolymer. Considering the contrast matching that occurs near $284 \mathrm{eV}$, where contrast between PE and PS is the same as the contrast between PE and vacuum, the R-SoXS profiles at these energies would be expected to have a peak at a similar location to that of the pure SES SAXS profile, which indeed they do. Inspection of the profile at $284.2 \mathrm{eV}$ reveals both a broad region of increased intensity at low q from the PS:vacuum contrast and a well-defined peak from the PS:PE contrast corresponding to a spacing of $38.8 \mathrm{~nm}$, consistent with the measured block spacing.

The contrast-matched R-SoXS used to characterize these mesoporous membranes demonstrates one of the biggest strengths of R-SoXS: its tunable contrast. SAXS could not detect the distance between the PS blocks, except in the case of a pure SES melt, but R-SoXS could detect this aspect of the morphology when the sample was probed with X-rays of the correct resonant excitation energy. Contrast-matched R-SoXS has also been used to elucidate other complex BCP morphologies [48]. In general, R-SoXS profiles acquired at several energies, carefully chosen based on the known contrast functions of the components in a sample, can elucidate complex morphologies.

\subsection{Organic Photovoltaics}

Numerous R-SoXS studies assessing spatial frequency distributions, relative composition variations or molecular orientation correlations have proven to be useful and relevant to understanding device performance in organic photovoltaics (OPVs) (e.g. [35, 45, 50, 59, 60, 8284]). Relative domain purity, as inferred from composition variations and use of some model

assumptions, is a relatively unique and recent development and has been shown to be relevant to understanding OPV structure-function relations [35, 69, 70]. Recent research, for example, by Mukherjee et al. [84] demonstrated this is the case in solution-processed small molecule solar cells. In this study, devices employing (p-DTS(FBTT $\left.)_{2}\right)$ as a donor molecule and [6,6]-phenyl$\mathrm{C}_{71}$-butyric acid methyl ester $\left(\mathrm{PC}_{71} \mathrm{BM}\right)$ as an acceptor were cast from chlorobenzene $(\mathrm{CB})$ with varying volume percentage of the additive 1,8-diiodooctane (DIO) and all other processing parameters left unchanged. 

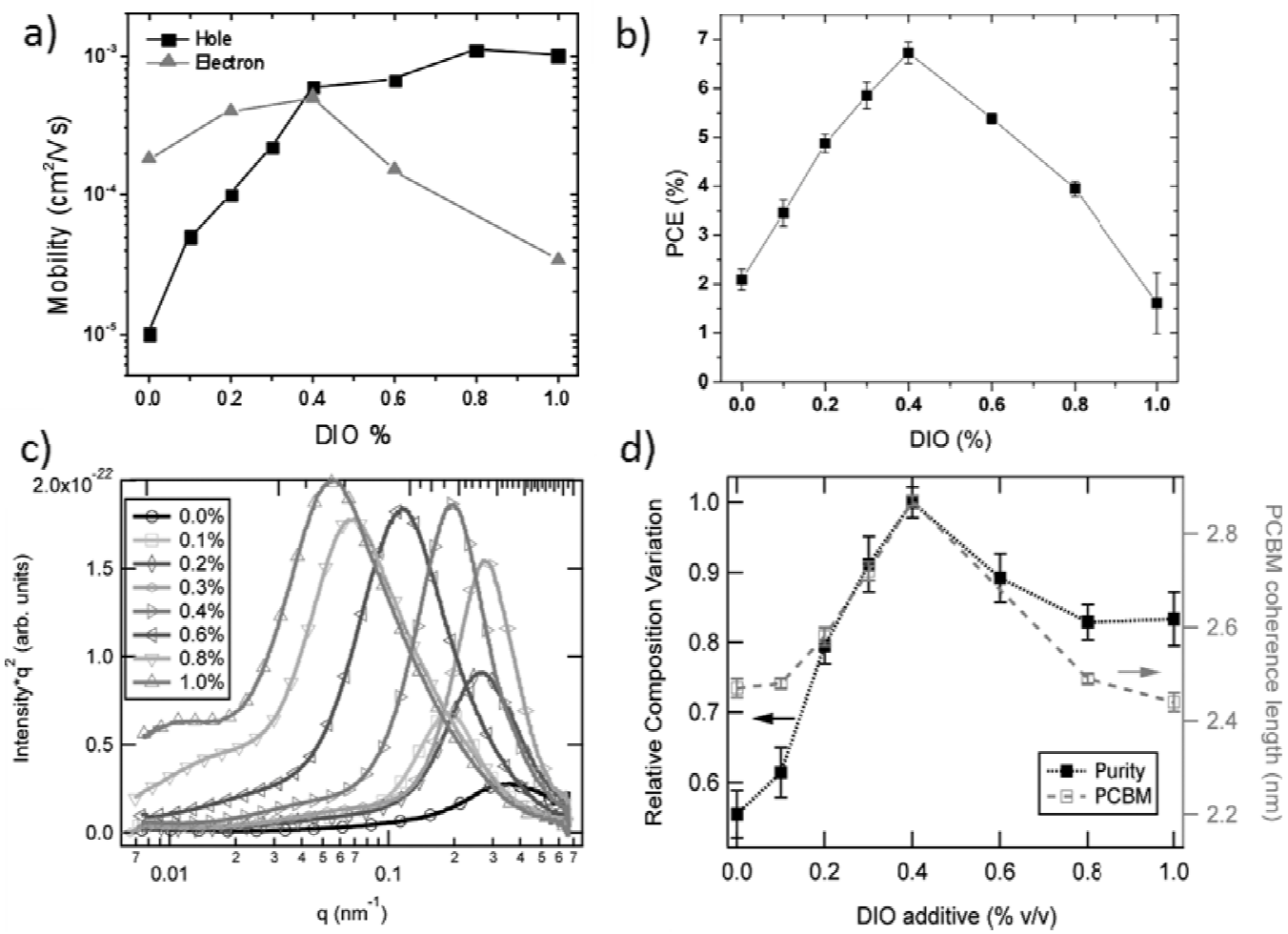

Fig. 5. Average device measurements are plotted vs. [DIO] in a) (hole and electron mobility) and b) (power conversion efficiency). In c), Lorentz corrected R-SoXS profiles normalized to scattering volume for varying [DIO] are shown. The relative composition variation extracted from c) is overplotted with the PCBM coherence length, extracted from GIWAXS measurements, vs [DIO] in d). Figure adapted from [84] Copyright Wiley-VCH Verlag $\mathrm{GmbH} \& \mathrm{Co} . \mathrm{KGaA}$. Reproduced with permission.

Fig. 5 a) and b) show the effect of changing DIO concentration ([DIO]) on charge carrier mobility and power conversion efficiency (PCE), respectively. Both mobility and PCE are positively correlated with [DIO] up to $0.4 \%$, whereas at higher [DIO] both the electron mobility and PCE are negatively correlated. R-SoXS profiles of the devices with varying [DIO] are shown in Fig. 5 c). From these profiles, relative composition variation is calculated (see section 3.2.) and plotted vs $[\mathrm{DIO}]$ in Fig. 5 d), in which a trend very similar to that displayed by the PCE in Fig. 5 b) is evident. The $\pi-\pi$ coherence length within $\mathrm{PC}_{71} \mathrm{BM}$ agglomerates, calculated from GIWAXS data, is also plotted vs [DIO] in Fig. 5 d) and exhibits a similar trend to both relative domain purity and PCE. A similar relation between crystal size and composition correlations as seen by R-SoXS was first observed by Ma et al. [59].

Evidently, device performance in (p-DTS(FBTT $\left.2_{2}\right): \mathrm{PC}_{71} \mathrm{BM}$ solar cells is determined by multiple factors, some of which seem to be competing. As has been shown for other OPVs, $\pi-\pi$ stacking correlates with improved charge transport $[85,86]$ and relative domain purity is important to device PCE [35]. The complex interrelationships observed between different aspects of morphology and device performance emphasize how R-SoXS can complement other morphological characterization techniques such as GIWAXS. Also, as R-SoXS is the only technique that can provide quantitative assessments of the average composition variations and 
therefore inferred average domain purity at these length scales, many of the insights into the morphology of these OPV devices would not be possible without it. It is largely because of these unique insights that studies of OPV active layers have been one of the most popular applications of R-SoXS.

The popularity of R-SoXS to study OPV morphology has been extended by Tumbleston et al. [50] to establish the importance of the degree of molecular ordering of several donor polymers in polymer:fullerene $\mathrm{BHJ}$ solar cells. The films were cast using $\mathrm{PC}_{61} \mathrm{BM}$ as an acceptor from several different solvents with and without fluorine substitution on the polymer backbones. The polymers used were PNDT-DTBT, PBnDT-DTBT, and PBnDT-TAZ.

We focus the discussion here on the devices employing PNDT-DTBT as they had the largest variety of processing conditions, being processed with and without fluorine substitution from both $\mathrm{CB}$ and dichlorobenzene (DCB). Correlation analysis of $\mathrm{J}_{\mathrm{sc}}$ and FF versus domain size, relative composition variations, and DMO for the different processing conditions, exhibited, positive correlations only between $\mathrm{J}_{\mathrm{sc}}$ and DMO as well as between FF and DMO. The Pearson coefficient, $\mathrm{r}$, for $\mathrm{J}_{\mathrm{sc}}$ versus $\mathrm{DMO}$ is greater than 0.95 , signifying an almost perfect linear relationship. Similarly, no correlation is found between $\mathrm{J}_{\mathrm{sc}}$ or FF and morphological parameters from GIWAXS. The authors attempted to qualitatively explain device performance using some combination of parameters besides the DMO and were unable to provide one that is not contradictory. Though the devices with PBnDT-DTBT and PBnDT-TAZ as a donor polymer do not have enough data points to demonstrate such a strong correlation, they display similar trends

to the PNDT-DTBT devices. Therefore, Tumbleston et al. concluded that the DMO is likely a critical morphological parameter for polymer:fullerene solar cells. More generally, since the molecular orientation at donor-acceptor interfaces has been demonstrated both theoretically [87, $88]$ and experimentally $[88,89]$ to be important to the photovoltaic process in small molecule devices, the DMO is likely an important measure in a broad range of organic solar cells. As PSoXS is currently the only characterization technique that can probe organic molecular orientation in complex 3-Dimensional morphologies, it will remain a key technique for future research into many organic systems.

\subsection{Organic Thin Film Transistors}

A 2012 study by Collins et al. [45] uses P-SoXS to study molecular ordering in organic thin film transistors (OTFTs). The OTFTs investigated employed the configuration shown in Fig. 6 e) with the polymer poly(2,5-bis(3-alkylthiophen-2-yl)thieno[3,2-b]thiophene (PBTTT) as the semiconducting layer and a dielectric layer made of poly(methyl methacrylate) (PMMA). The PBTTT layer was fabricated separately and then laminated atop the PMMA. Devices were subjected to one of four annealing processes. Either the PBTTT layer was pre-annealed before laminating, the bilayer was annealed after laminating (post-annealed), the PBTTT was preannealed and the bilayer was also annealed (pre- and post-annealed), or there was no annealing (as-cast). The temperature was chosen to anneal in the liquid crystalline phase of PBTTT [90]. P-SoXS and STXM were used to study the effects of the various annealing methods on the 
morphology of the liquid crystalline PBTTT films which were then correlated to OTFT performance.

a)

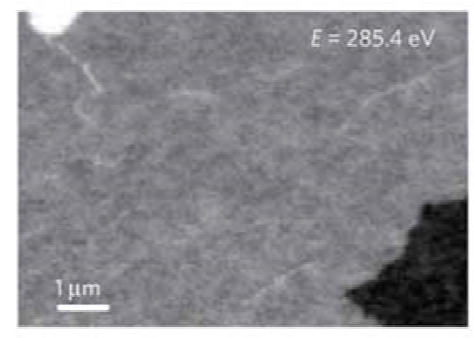

b)



c)



d)

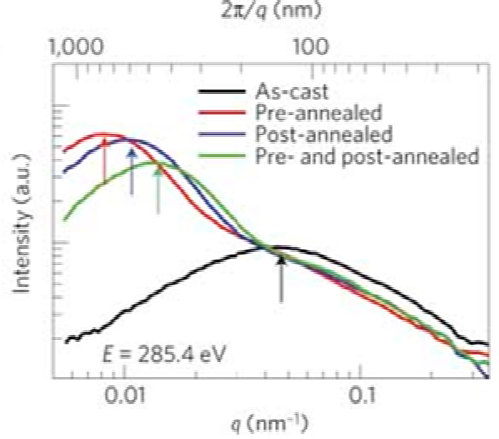

e)



f)

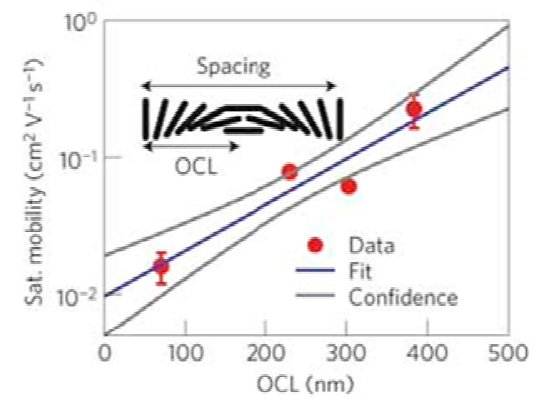

Fig. 6. a) and b) show STXM images at the PBTTT C-1s $\rightarrow \pi_{\mathrm{C}=\mathrm{C}}^{*}$ resonant energy $(285.4 \mathrm{eV})$ for as cast and postannealed PBTTT/PMMA bilayers, respectively. 1-Dimensional R-SoXS profiles obtained by azimuthal integration of the 2-Dimensional scattering data for different processing conditions are shown in c) and d). The profiles in c) are taken below resonance $(250 \mathrm{eV})$, while those in d) are taken at $285.4 \mathrm{eV}$. The OCL extracted from domain spacings measured in d) are shown with an exponential fit in f). Also in f) is an illustration of how the OCL should be half of the measured domain spacings from d). In e) is a schematic of the OTFT. This figure was reprinted by permission from Macmillan Publishers Ltd: Nature Materials [45], copyright 2012.

STXM images of the as-cast and post-annealed films acquired at $285.4 \mathrm{eV}$ are shown in Fig. 6 a) and b), respectively. At this energy the $\mathrm{C}-1 \mathrm{~s} \rightarrow \pi_{\mathrm{C}=\mathrm{C}}^{*}$ transition in PBTTT occurs while absorption from PMMA is negligible. Therefore, any domains observed at this energy that are not also observed at non-resonant energies must be orientational structure within the PBTTT. The as-cast film shows some orientational structure, though with weak contrast, and the postannealed film has clear orientational domains present with much larger contrast. While quantitative analysis of orientational domains is possible with STXM [91, 92], in this case they are either too small or have too little contrast.

In contrast, P-SoXS allows for the sizes of these orientational domains to be quantified readily. The OCL, defined in section 4 above, is a measure of the length scale over which an orientational domain in a liquid crystalline material remains orientationally correlated. The connection between OCL and domain spacings measured by P-SoXS (or STXM) is illustrated by the schematic in the inset of Fig. $6 \mathrm{f}$ ). Due to the liquid crystalline nature of the PBTTT film, local molecular orientation varies relatively continuously. Since the dominant feature in the P- 
SoXS profiles is due to orientational contrast, this means that they are measuring the spatial frequency that corresponds to the spacing between points of similar orientation separated by a region of less similar orientation. Therefore the OCL of each film is said to be half the spacing measured by P-SoXS. The OCL is highly relevant to charge transport in OTFTs, as in many cases both intra- and interchain charge transport are highly dependent on the direction along or between molecules [85, 93, 94].

P-SoXS profiles, shown in Fig. 6 c) and d), allowed for a quantitative assessment of the OCL in the PBTTT films. The profiles in Fig. 6 c) were acquired below resonance, at $250 \mathrm{eV}$, and are therefore indicative of surface roughness or density fluctuations in the films. The observed features in these profiles are relatively similar between annealing methods and show no correlation to OTFT performance, as quantified by the saturation field-effect hole mobility. On the other hand, mobilities were highly correlated ( $r>0.94)$ with OCLs extracted from the profiles in Fig. 6 d), which were acquired at resonance $(285.4 \mathrm{eV})$. The data, the exponential fit, and 68\% confidence interval are shown in Fig. 6 f).

It is clear from the correlation between mobility and OCL that, as expected, the OCL is critically important to charge transport in this system. In this case, the superior spatial resolution and significantly higher signal to noise ratio of P-SoXS, compared to STXM as well TEM, along with its orientational contrast, allowed OCLs to be quantified and correlated with OTFT performance. For any system with orientational ordering but limited means of contrast between orientational domains, such as the pure liquid crystalline PBTTT film studied here, P-SoXS has proven to be a decidedly useful tool.

\section{Emerging Applications with New Geometries and Controlled Sample Environments.}

New extensions of R-SoXS continue to be developed. One such extension is R-SoXS from samples that are manipulated in-situ (thermally, mechanically, electrically, etc.). A recent study by Zhu et al. used P-SoXS to study the ordering of liquid crystalline organic nanofilaments at varying temperature [95]. 

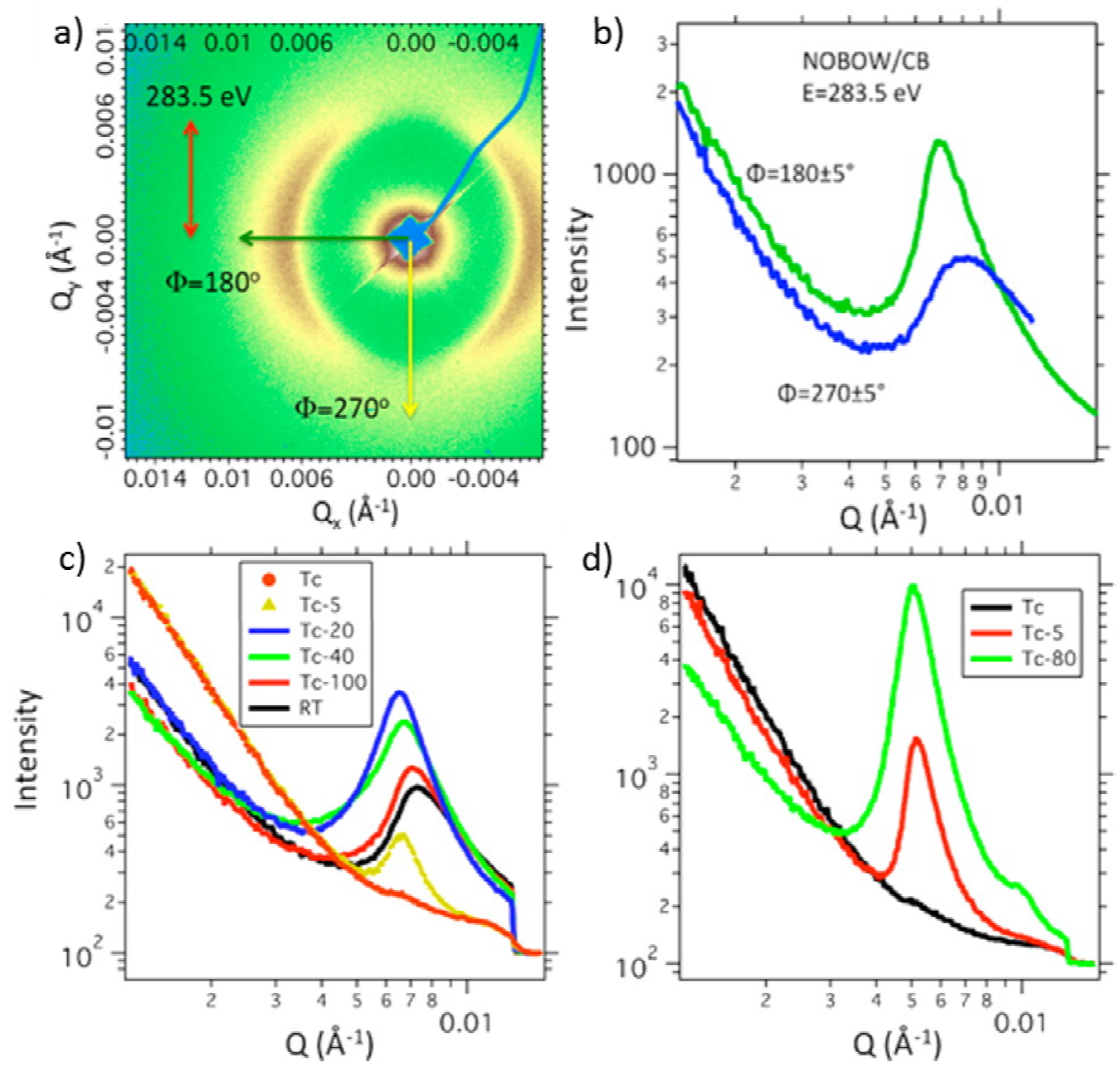

Fig. 7. a) A scattering pattern collected at $283.5 \mathrm{eV}$, with red arrows indicating the direction of the polarization vector of the incoming radiation. Yellow and green arrows indicate the vertical and horizontal directions, respectively, for which the 1-D profiles are given in b). The temperature dependence of the scattering pattern upon heating is shown in b) and upon cooling in d). Note that the 1-D profiles in b), c), and d) have been Lorentz corrected, such that the ordinate axis is $\mathrm{I}(\mathrm{q}) * \mathrm{q}^{2}$. Figure adapted with permission from [95]. Copyright 2015 American Chemical Society.

The nanofilaments have a helical structure, the half pitch of which results in a distinct PSoXS feature as seen in Fig. 7 a). For reasons discussed in the text, the horizontal profile is taken to represent the half-pitch and thus the ordering of the filament as a function of temperature. In Fig. 7 c) the horizontal R-SoXS profiles of the filaments as temperature is increased from room temperature to above a temperature at which the material becomes isotropic (and therefore the scattering peak disappears). The changing location of the peak indicates that the half-pitch changes upon heating. Interestingly, after the sample is heated to the isotropic phase and subsequently cooled, the peak location remains constant as shown in Fig. 7 d). The authors take this as evidence that the ordering of the filaments are not optimal upon casting and only reach an equilibrium after annealing and subsequent cooling. 
The nanofilaments in this study could be utilized in photovoltaics or in chemical synthesis applications, but in-situ sample manipulation in conjunction with R-SoXS will allow for many more applications. In one such example, Young et al. used R-SoXS to determine the feasibility of a solid polymer electrolyte blend for use in high performance rechargeable batteries [96]. The batteries would require the blend to maintain temperatures above $116^{\circ} \mathrm{C}$ during use.

Other extensions of R-SoXS that are being developed make use of different scattering geometries than standard transmission R-SoXS, such as grazing R-SoXS (GR-SoXS) [97, 98]. In a GR-SoXS experiment, the sample is oriented only a few degrees away from parallel to the incident beam. An approximate $\theta-2 \theta$ geometry is maintained, though this requirement is not as strict as in reflectivity experiments because, in GR-SoXS, the diffuse scattering pattern is measured with an area detector. The analysis of this scattering can quantify both in and out of plane morphological parameters and so GR-SoXS is very well suited for the study of multilayer organic devices such as OTFTs. Similar to that of R-SoXR [77, 99-101], the sensitivity of GRSoXS to specific layers can be controlled via contrast matching. Also, varying $\theta$ below the critical angle of a layer allows for a means to control the beam penetration depth within layers. Diffuse R-SoXS scattering has already been extended to higher angles in order to probe smaller structures [56] and, as always with resonant soft X-rays, local molecular ordering can influence the observed scatter and so could, in principle, be quantified. We note that as the effective film thickness is much higher for GR-SoXS than for standard transmission R-SoXS, new theoretical approaches must be used such as the distorted wave Born approximation as well as new, often simulation-intensive, analysis methods, which will present new challenges to the R-SoXS community.
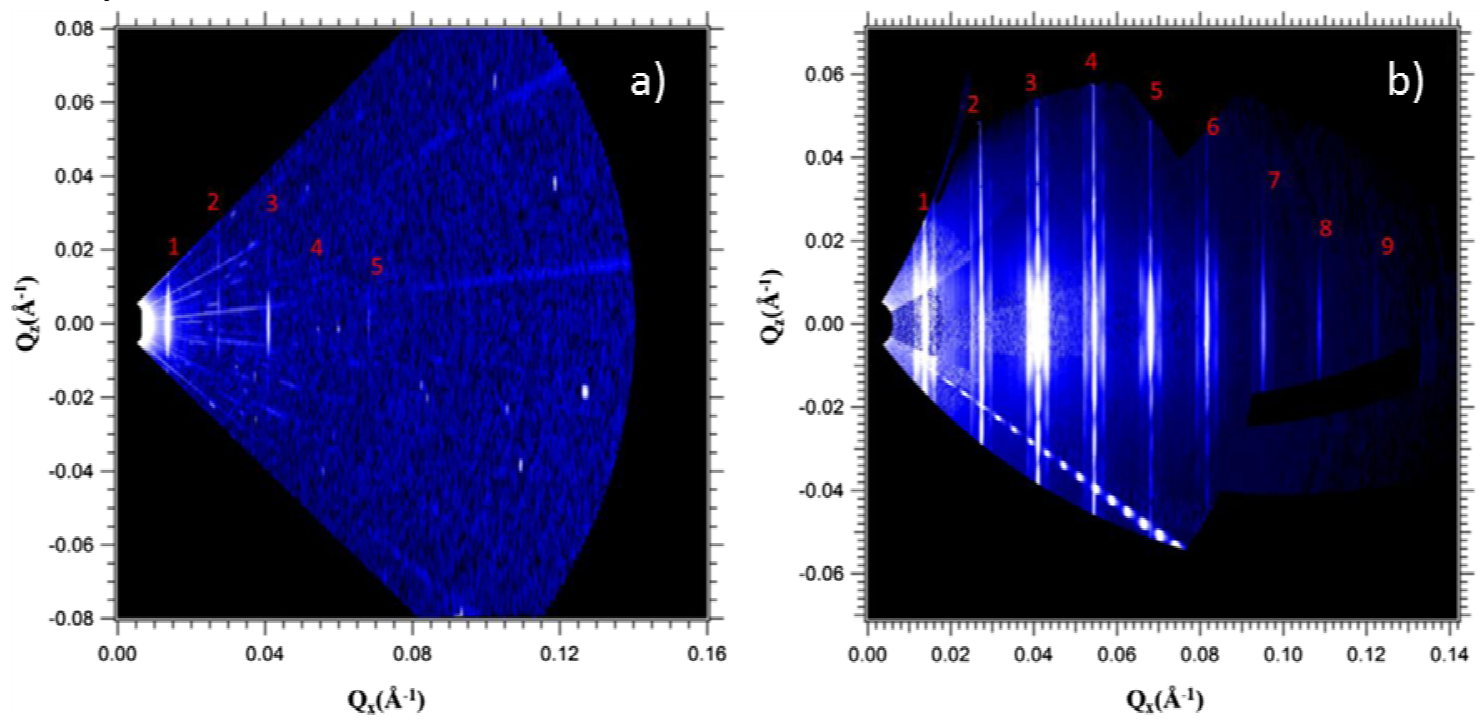

Fig. 8. 2-Dimensional Intensity maps characteristic of a 3-Dimensional grating structure. In a) the data was acquired with hard X-rays $(17 \mathrm{keV})$, while in b) resonant soft X-rays were used. Figure borrowed from [102] with permission from SPIE and the author.

Another emerging R-SoXS technique is resonant critical-dimension small angle X-ray scattering (res-CDSAXS) [102]. Although clearly an extension of R-SoXS methodology, the 
naming chosen emphasizes connection to prior non-resonant CDSAXS efforts [103], emphasizing a specific application undertaken to characterize the morphology of nanoscale gratings. A res-CDSAXS experiment begins with a similar transmission geometry to standard RSoXS, with an area detector recording the scattering pattern at multiple locations to extend the spatial frequency range. The sample is subsequently rotated in steps relative to the incoming beam and new scattering data are recorded at each step. Combining the different 2-Dimensional data from various angles of the sample relative to the beam into an intensity vs q map, as seen in Fig. 8 for a PS-b-PMMA BCP grating membrane, allows for the average 3-D structure of the grating to be reconstructed. The high intensity, high quality data of res-CDSAXS allows for superior reconstructions (Fig. 8 b)). Though res-CDSAXS is particularly well suited for gratings of block copolymers due to their highly regular and periodic structure, the same principles could potentially be applied to study 3-D structure in applications of organic materials that are typically much more disordered, such as OPVs.

In a recent study by Ingham et al., R-SoXS was used to study aggregation in milk, a biological system extensively studied previously with a number of techni ${ }^{\mathrm{q}}$ ues including conventional SAX and SANS [104]. Transmission R-SoXS at the calcium $\mathrm{L}_{2} / \mathrm{L}_{3}$ absorption edge $(\sim 540 \mathrm{eV})$ was used to help clarify the structure of the various proteins that make up milk. Previously these structures were not fully understood, as SAXS could not unambiguously attribute scattering peaks to specific aspects of the aggregates.
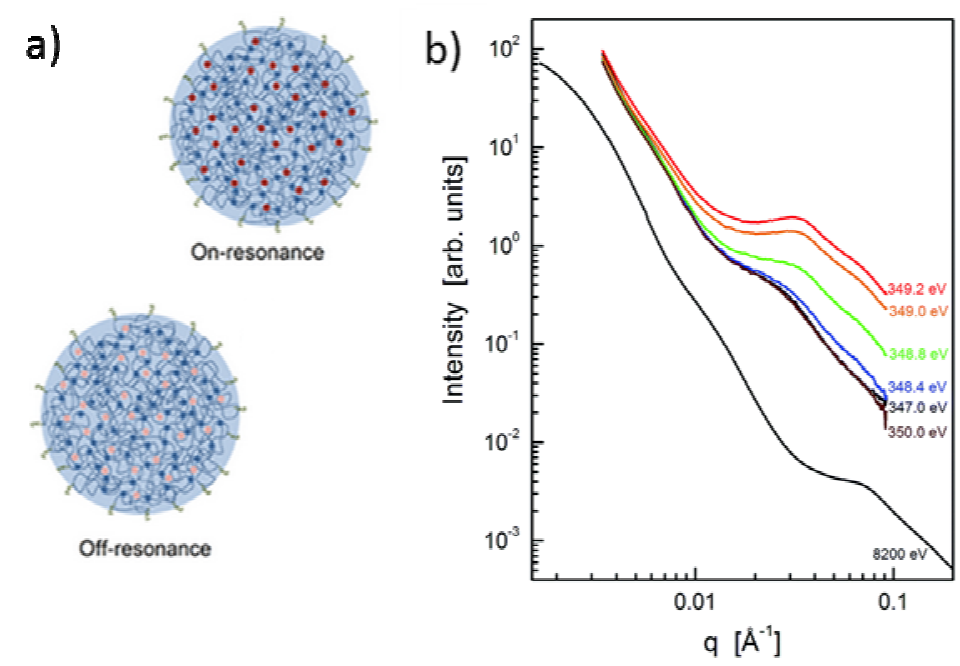

Fig. 9. a) is a cartoon demonstrating how the colloidal calcium phosphate spheres contrast varies on- vs offresonance. b) features 1-D profiles at resonant and non-resonant energies including $8200 \mathrm{eV}$ (SAXS). This figure is adapted with permission from [104]. Copyright 2015 American Chemical Society.

One of the main questions regarding previously reported SAXS data was the cause of the scattering peak located near $0.33 \AA^{-1}$ [105]. As shown in Fig. 9 b), the presence of this peak is clearly dictated by the choice of energy to be on or off of the calcium resonance. Thus the 
authors are able to determine that this spacing is characteristic of the interparticle spacing for colloidal calcium phosphate spheres.

This work is unique among studies that have utilized R-SoXS for several reasons. It is the first study that collected R-SoXS at the calcium $\mathrm{L}_{2} / \mathrm{L}_{3}$ edge, though other studies have used other absorption edges besides the carbon K-edge such as the oxygen K-edge [106]. Also, though wet cells have been studied with R-SoXS previously [101], their involvement in R-SoXS studies has been somewhat infrequent. Additionally, to the best of our knowledge, this is the first instance of R-SoXS being used to study strictly biological samples.

\section{Summary and Conclusions}

R-SoXS techniques are able to provide unique quantitative measurements in organic systems at length scales from several nanometers to several microns. They exploit scattering contrast due to highly oriented, highly energy dependent electronic state transitions. Scattering contrast between organic materials that are chemically very similar, as well as different orientations of the same material, can be controlled by simply changing the excitation energy by a few $\mathrm{eV}$. The distribution of spatial frequencies (at a given contrast) is easily obtained from the measured 2-Dimensional scattering data and converted to real space domain spacings if a sample with approximately equal volume fractions of its constituents is analyzed. R-SoXS intensities, integrated over spatial frequency probed, can be used to compare relative average purity of all domains and, for anisotropic scattering data due to local correlations in molecular ordering, to ascertain the average local DMO.

Recent and future innovations in R-SoXS techniques show great promise in expanding the types of morphological information that can be obtained as well as the types of samples that can be studied with R-SoXS. Modified experimental designs will allow for new capabilities, such as in-situ sample manipulation, or new classes of samples, such as wet cells, that will bring the quantitative power of R-SoXS to bear in novel applications. Techniques that utilize new sample-detector scattering geometries will allow sample morphology to be characterized in new ways that are highly relevant to technological applications. For example, GR-SoXS can quantify out of plane structure with controllable depth sensitivity, which makes it highly appealing for the study of OTFTs, and res-CDSAXS can provide information about the average 3-Dimensional structure in a material, which is particularly relevant to production of nanoscale gratings. These novel implementations of R-SoXS make use of its unparalleled capabilities and ensure that it will continue to be an essential tool for characterizing the morphology of organic materials.

Currently, most applications of R-SoXS the majority of characterization work on soft matter systems using R-SoXS is done at beamline 11.0.2.1 at the Advanced Light Source. Construction of this beamline was completed in 2011 [76]. The productivity of this facility is rapidly rising, with the number of publications approximately doubling every year: In 2011, beamline 11.0.2.1 produced 5 publications (3 in DOE high-profile publications); in 2012, 12 (3); in 2013, 20 (7); and in 2014, 44 (18). The capacity of this facility is limited by sharing an 
undulator with another end station and the facility is consequently significantly oversubscribed. We believe there is a great opportunity for other synchrotron radiation facilities to build dedicated soft matter R-SoXS facilities to provide additional capacity and develop new capabilities. We hope that the exemplary applications and future outlook presented here further grow the user community and make a compelling case for additional facilities.

Acknowledgement: JHC and AH supported by DOE, OS, BES, Division of Materials Science and Engineering under Contract DE-FG02-98ER45737, and ONR grant N000141410531. We gratefully acknowledge fruitful discussions with Eliot Gann and Subhrangsu Mukherjee as well as work done by Maolong Tang that helped further our understanding of R-SoXS. 
References

1. Chiang, C.K., et al., Electrical-Conductivity in Doped Polyacetylene. Physical Review Letters, 1977. 39(17): p. 1098-1101.

2. Novoselov, K.S., et al., Electric field effect in atomically thin carbon films. Science, 2004. 306(5696): p. 666-669.

3. Chen, S.S., et al., Thermal conductivity of isotopically modified graphene. Nature Materials, 2012. 11(3): p. 203-207.

4. Lee, C., et al., Measurement of the elastic properties and intrinsic strength of monolayer graphene. Science, 2008. 321(5887): p. 385-388.

5. Mitchell, G.E., et al., Molecular bond selective $x$-ray scattering for nanoscale analysis of soft matter. Applied Physics Letters, 2006. 89(4).

6. Araki, T., et al., Resonant soft x-ray scattering from structured polymer nanoparticles. Applied Physics Letters, 2006. 89(12): p. 124106.

7. Wetch, C.F., et al., Contrast variation using resonant soft $X$-ray scattering to probe structure of polymer thin films. Abstracts of Papers of the American Chemical Society, 2005. 230: p. U3592U3593.

8. Stöhr, J., NEXAFS spectroscopy. Springer series in surface sciences. 1992, Berlin ; New York: Springer-Verlag. $x v, 403 p$.

9. Dhez, O., H. Ade, and S.G. Urquhart, Calibrated NEXAFS spectra of some common polymers. Journal of Electron Spectroscopy and Related Phenomena, 2003. 128(1): p. 85-96.

10. Urquhart, S.G. and H. Ade, Trends in the Carbonyl Core (C 1S, O 1S) $\rightarrow \pi^{*} C=$ OTransition in the Near-Edge X-ray Absorption Fine Structure Spectra of Organic Molecules. The Journal of Physical Chemistry B, 2002. 106(34): p. 8531-8538.

11. Watts, B., et al., Calibrated NEXAFS spectra of common conjugated polymers. J Chem Phys, 2011. 134(2): p. 024702.

12. Fink, J., et al., Resonant elastic soft x-ray scattering. Reports on Progress in Physics, 2013. 76(5).

13. Hannon, J.P., et al., X-Ray Resonance Exchange Scattering. Physical Review Letters, 1988. 61(10): p. $1245-1248$.

14. Characterization analysis of polymers. 2008, Hoboken, N.J.: Wiley-Interscience. $x, 977$ p.

15. Horowitz, P., Scanning X-Ray Microscope Using Synchrotron Radiation. Science, 1972. 178(4061): p. 608-\&.

16. Ade, H. and A.P. Hitchcock, NEXAFS microscopy and resonant scattering: Composition and orientation probed in real and reciprocal space. Polymer, 2008. 49(3): p. 643-675.

17. Ade, H., et al., Chemical Contrast in X-Ray Microscopy and Spatially Resolved Xanes Spectroscopy of Organic Specimens. Science, 1992. 258(5084): p. 972-975.

18. Ade, H. and B. Hsiao, X-ray Linear Dichroism Microscopy. Science, 1993. 262(5138): p. 14271429.

19. Watts, B. and C.R. McNeill, Simultaneous Surface and Bulk Imaging of Polymer Blends with X-ray Spectromicroscopy. Macromolecular Rapid Communications, 2010. 31(19): p. 1706-1712.

20. Hub, C., et al., Surface sensitivity in scanning transmission $x$-ray microspectroscopy using secondary electron detection. Rev Sci Instrum, 2010. 81(3): p. 033704.

21. Ade, H., et al., Bulk and surface characterization of a dewetting thin film polymer bilayer. Applied Physics Letters, 1998. 73(25): p. 3775-3777.

22. Sham, T.-K., Chemical applications of synchrotron radiation. Advanced series in physical chemistry. 2002, New Jersey: World Scientific. 2 v. (xiii, 1227 p.).

23. Kilcoyne, A.L.D., et al., Interferometer-controlled scanning transmission $X$-ray microscopes at the Advanced Light Source. Journal of Synchrotron Radiation, 2003. 10(2): p. 125-136. 
24. Collins, B.A. and H. Ade, Quantitative compositional analysis of organic thin films using transmission NEXAFS spectroscopy in an X-ray microscope. Journal of Electron Spectroscopy and Related Phenomena, 2012. 185(5-7): p. 119-128.

25. Coffey, T., S.G. Urquhart, and H. Ade, Characterization of the effects of soft X-ray irradiation on polymers. Journal of Electron Spectroscopy and Related Phenomena, 2002. 122(1): p. 65-78.

26. Rightor, E.G., et al., Spectromicroscopy of poly(ethylene terephthalate): Comparison of spectra and radiation damage rates in $x$-ray absorption and electron energy loss. Journal of Physical Chemistry B, 1997. 101(11): p. 1950-1960.

27. Wang, J., et al., Radiation damage in soft $X$-ray microscopy. Journal of Electron Spectroscopy and Related Phenomena, 2009. 170(1-3): p. 25-36.

28. Obst, M., J. Wang, and A.P. Hitchcock, Soft X-ray spectro-tomography study of cyanobacterial biomineral nucleation. Geobiology, 2009. 7(5): p. 577-591.

29. Johansson, G.A., et al., Three-dimensional chemical mapping by scanning transmission X-ray spectromicroscopy. Journal of Synchrotron Radiation, 2007. 14: p. 395-402.

30. Collins, B.A., J.R. Tumbleston, and H. Ade, Miscibility, Crystallinity, and Phase Development in P3HT/PCBM Solar Cells: Toward an Enlightened Understanding of Device Morphology and Stability. Journal of Physical Chemistry Letters, 2011. 2(24): p. 3135-3145.

31. Giewekemeyer, K., et al., Ptychographic coherent $x$-ray diffractive imaging in the water window. Optics Express, 2011. 19(2): p. 1037-1050.

32. Thibault, P., et al., High-resolution scanning x-ray diffraction microscopy. Science, 2008. 321(5887): p. 379-382.

33. Marchesini, S., et al., Massively parallel X-ray holography. Nature Photonics, 2008. 2(9): p. 560 563.

34. Hitchcock, A., Journal of Electron Spectroscopy and Related Phenomena, 2015. 200.

35. Collins, B.A., et al., Absolute measurement of domain composition and nanoscale size distribution explains performance in PTB7: PC71BM Solar Cells. Advanced Energy Materials, 2013. 3(1): p. 65-74.

36. Wendin, G., Anomalous X-Ray-Scattering. Physica Scripta, 1980. 21(3-4): p. 535-542.

37. Goerigk, G., et al., Anomalous small-angle $X$-ray scattering in materials science. Journal of Applied Crystallography, 2003. 36(1): p. 425-429.

38. Phillips, J.C., et al., Liii-Edge Anomalous X-Ray-Scattering by Cesium Measured with Synchrotron Radiation. Science, 1978. 201(4352): p. 257-259.

39. Bremer, J., Anomalous Scattering of X-Ray Photons at the $K$ Absorption-Edge of Free Atoms and Molecules, Calculated by Means of the Modified Kramers-Heisenberg Dispersion Formula. Journal of Physics B-Atomic Molecular and Optical Physics, 1979. 12(17): p. 2797-2807.

40. Templeton, D.H. and L.K. Templeton, Polarized X-Ray Absorption and Double-Refraction in Vanadyl Bisacetylacetonate. Acta Crystallographica Section A, 1980. 36(Mar): p. 237-241.

41. Kronig, R.D.L., On the theory of dispersion of $x$-rays. Journal of the Optical Society of America and Review of Scientific Instruments, 1926. 12(6): p. 547-557.

42. Jackson, J.D., Classical electrodynamics. 3. ed. 1999, New York: John Wiley. xxi, $808 \mathrm{s.}$

43. Attwood, D.T., Soft $x$-rays and extreme ultraviolet radiation : principles and applications. 2000, Cambridge ; New York: Cambridge University Press. xvi, 470 p.

44. Yan, H.P., et al., Accurate and Facile Determination of the Index of Refraction of Organic Thin Films Near the Carbon 1s Absorption Edge. Physical Review Letters, 2013. 110(17).

45. Collins, B.A., et al., Polarized X-ray scattering reveals non-crystalline orientational ordering in organic films. Nat Mater, 2012. 11(6): p. 536-543.

46. Gann, E.H., Using Resonant Soft X-rays to Reveal Internal Organic Thin Film Structure. 2013, [Raleigh, North Carolina]: North Carolina State University. 1 online resource (xi, 164 pages). 
47. Gann, E.H., et al. 2015.

48. Wang, C., et al., Defining the nanostructured morphology of triblock copolymers using resonant soft X-ray scattering. Nano Lett, 2011. 11(9): p. 3906-3911.

49. Wong, D.T., et al., Mesoporous Block Copolymer Morphology Studied by Contrast-Matched Resonant Soft X-ray Scattering. Macromolecules, 2012. 45(22): p. 9188-9195.

50. Tumbleston, J.R., et al., The influence of molecular orientation on organic bulk heterojunction solar cells. Nature Photonics, 2014. 8(5): p. 385-391.

51. Gu, Y., et al., Guided crystallization of P3HT in ternary blend solar cell based on P3HT:PCPDTBT:PCBM. Energy \& Environmental Science, 2014. 7(11): p. 3782-3790.

52. Ma, W., et al., Tuning Local Molecular Orientation-Composition Correlations in Binary Organic Thin Films by Solution Shearing. Advanced Functional Materials, 2015: p. n/a-n/a.

53. Watts, B., T. Schuettfort, and C.R. McNeill, Mapping of Domain Orientation and Molecular Order in Polycrystalline Semiconducting Polymer Films with Soft X-Ray Microscopy. Advanced Functional Materials, 2011. 21(6): p. 1122-1131.

54. Mezger, M., et al., Molecular orientation in soft matter thin films studied by resonant soft $x$-ray reflectivity. Physical Review B, 2011. 83(15).

55. Pasquali, L., et al., Structural and electronic properties of anisotropic ultrathin organic films from dichroic resonant soft $x$-ray reflectivity</article-title>. Physical Review B, 2014. 89(4): p. 045401.

56. Stone, K.H. and J.B. Kortright, Molecular anisotropy effects in carbon K-edge scattering: Depolarized diffuse scattering and optical anisotropy. Physical Review B, 2014. 90(10).

57. Mach, P., et al., Structures of chiral smectic-C mesophases revealed by polarization-analyzed resonant $x$-ray scattering. Physical Review E, 1999. 60(6): p. 6793-6802.

58. Stribeck, N., X-ray scattering of soft matter, in Springer laboratory. 2007, Springer,: Berlin ; New York. p. xx, 238 p. ill. (some col.).

59. Ma, W., et al., Quantification of nano- and mesoscale phase separation and relation to donor and acceptor quantum efficiency, J(sc), and FF in polymer:fullerene solar cells. Adv Mater, 2014. 26(25): p. 4234-4241.

60. Love, J.A., et al., Interplay of Solvent Additive Concentration and Active Layer Thickness on the Performance of Small Molecule Solar Cells. Advanced Materials, 2014: p. n/a-n/a.

61. Ferdous, S., et al., Solvent-Polarity-Induced Active Layer Morphology Control in Crystalline Diketopyrrolopyrrole-Based Low Band Gap Polymer Photovoltaics. Advanced Energy Materials, 2014. 4(2).

62. Brumberger, H., Modern aspects of small-angle scattering. NATO ASI series Series C, Mathematical and physical sciences. 1995, Dordrecht ; Boston: Kluwer Academic Publishers. xv, $463 \mathrm{p}$.

63. Cruz, C.S., et al., Novel Aspects in the Structure of Poly(Ethylene-Terephthalate) as Revealed by Means of Small-Angle X-Ray-Scattering. Macromolecules, 1991. 24(22): p. 5980-5990.

64. Tchoubar, D. and J. Mering, Interpretation of Central Scattering of X-Rays by Porous Systems .2. Examples of Application. Journal of Applied Crystallography, 1969. 2: p. 128-\&.

65. Mering, P.J. and D. Tchoubar, Interpretation of Central Diffusion of X-Rays by Porous Systems .I. Journal of Applied Crystallography, 1968. 1: p. 153-\&.

66. Mering, J. and Tchoubar.D, Diffusion Centrale Des Rayons X Par Des Suspensions Diluees De Particules . Determination De La Distribution Des Cordes. Comptes Rendus Hebdomadaires Des Seances De L Academie Des Sciences, 1965. 261(16): p. 3096-\&.

67. Mering, J. and Tchoubar.D, Diffusion Centrale Des Rayons X Par Des Systemes Concentres. Comptes Rendus Hebdomadaires Des Seances De L Academie Des Sciences Serie B, 1966. 262(26): p. 1703-\&. 
68. Stribeck, N., Scattering of Soft Condensed Matter: From Fundaments to Application. Applications of Synchrotron Light to Scattering and Diffraction in Materials and Life Sciences, 2009. 776: $p$. 23-60.

69. Kesava, S.V., et al., Domain Compositions and Fullerene Aggregation Govern Charge Photogeneration in Polymer/Fullerene Solar Cells. Advanced Energy Materials, 2014. 4(11).

70. Tumbleston, J.R., et al., Morphology linked to miscibility in highly amorphous semi-conducting polymer/fullerene blends. Polymer, 2014. 55(19): p. 4884-4889.

71. Porod, G., Die Rontgenkleinwinkelstreuung Von Dichtgepackten Kolloiden Systemen .2. KolloidZeitschrift and Zeitschrift Fur Polymere, 1952. 125(2): p. 108-122.

72. Porod, G., Die Rontgenkleinwinkelstreuung Von Dichtgepackten Kolloiden Systemen .1. KolloidZeitschrift and Zeitschrift Fur Polymere, 1951. 124(2): p. 83-114.

73. Yin, W. and M. Dadmun, A New Model for the Morphology of P3HT/PCBM Organic Photovoltaics from Small-Angle Neutron Scattering: Rivers and Streams. Acs Nano, 2011. 5(6): p. 4756-4768.

74. Liu, F., et al., Relating Chemical Structure to Device Performance via Morphology Control in Diketopyrrolopyrrole-Based Low Band Gap Polymers. Journal of the American Chemical Society, 2013. 135(51): p. 19248-19259.

75. Smith, A.P. and H. Ade, Quantitative orientational analysis of a polymeric material (Kevlar ${ }^{\circledR}$ fibers) with x-ray microspectroscopy. Applied Physics Letters, 1996. 69(25): p. 3833.

76. Gann, E., et al., Soft x-ray scattering facility at the Advanced Light Source with real-time data processing and analysis. Rev Sci Instrum, 2012. 83(4): p. 045110.

77. Wang, C., T. Araki, and H. Ade, Soft $x$-ray resonant reflectivity of low-Z material thin films. Applied Physics Letters, 2005. 87(21): p. 214109.

78. Wang, C., et al., Interfacial widths of conjugated polymer bilayers. J Am Chem Soc, 2009. 131(35): p. 12538-12539.

79. Yan, H., et al., Influence of dielectric-dependent interfacial widths on device performance in topgate P(NDI2OD-T2) field-effect transistors. Applied Physics Letters, 2012. 101(9): p. 093308.

80. Nayak, M., et al., Determination of interlayer composition at buried interfaces using soft x-ray resonant reflectivity. Applied Physics Letters, 2006. 89(18).

81. Webster, J. and S. Langridge, Applications of index matching in reflectometry, SANS and Brewster angle microscopy. Current Opinion in Colloid \& Interface Science, 1999. 4(3): p. 186-189.

82. Tumbleston, J.R., et al., Fluorinated Polymer Yields High Organic Solar Cell Performance for a Wide Range of Morphologies. Advanced Functional Materials, 2013. 23(27): p. 3463-3470.

83. Ma, W., et al., Domain Purity, Miscibility, and Molecular Orientation at Donor/Acceptor Interfaces in High Performance Organic Solar Cells: Paths to Further Improvement. Advanced Energy Materials, 2013. 3(7): p. 864-872.

84. Mukherjee, S., et al., Importance of domain purity and molecular packing in efficient solutionprocessed small-molecule solar cells. Advanced Materials, 2014.

85. Steyrleuthner, R., et al., The role of regioregularity, crystallinity, and chain orientation on electron transport in a high-mobility n-type copolymer. J Am Chem Soc, 2014. 136(11): p. 42454256.

86. Li, W., et al., Mobility-Controlled Performance of Thick Solar Cells Based on Fluorinated Copolymers. Journal of the American Chemical Society, 2014. 136(44): p. 15566-15576.

87. Verlaak, S., et al., Electronic Structure and Geminate Pair Energetics at Organic-Organic Interfaces: The Case of Pentacene/C-60 Heterojunctions. Advanced Functional Materials, 2009. 19(23): p. 3809-3814.

88. Ojala, A., et al., Merocyanine/C60 Planar Heterojunction Solar Cells: Effect of Dye Orientation on Exciton Dissociation and Solar Cell Performance. Advanced Functional Materials, 2012. 22(1): p. 86-96. 
89. Rand, B.P., et al., The Impact of Molecular Orientation on the Photovoltaic Properties of a Phthalocyanine/Fullerene Heterojunction. Advanced Functional Materials, 2012. 22(14): p. 29872995.

90. Zhang, X.R., et al., In-Plane Liquid Crystalline Texture of High-Performance Thienothiophene Copolymer Thin Films. Advanced Functional Materials, 2010. 20(23): p. 4098-4106.

91. Brauer, B., et al., X-ray Microscopy Imaging of the Grain Orientation in a Pentacene Field-Effect Transistor. Chemistry of Materials, 2010. 22(12): p. 3693-3697.

92. Kline, R.J., et al., Significant dependence of morphology and charge carrier mobility on substrate surface chemistry in high performance polythiophene semiconductor films. Applied Physics Letters, 2007. 90(6).

93. Street, R.A., Unraveling Charge Transport in Conjugated Polymers. Science, 2013. 341(6150): p. 1072-1073.

94. Schubert, M., et al., Correlated Donor/Acceptor Crystal Orientation Controls Photocurrent Generation in All-Polymer Solar Cells. Advanced Functional Materials, 2014. 24(26): p. 40684081.

95. Zhu, C., et al., Probing and Controlling Liquid Crystal Helical Nanofilaments. Nano Letters, 2015.

96. Young, N.P., et al., Investigating polypropylene-poly(ethylene oxide)-polypropylene triblock copolymers as solid polymer electrolytes for lithium batteries. Solid State Ionics, 2014. 263: p. 87-94.

97. Ruderer, M.A., et al., Morphology and Optical Properties of P3HT:MEH-CN-PPV Blend Films. Macromolecules, 2013. 46(11): p. 4491-4501.

98. Gann, E., et al., Topographic measurement of buried thin-film interfaces using a grazing resonant soft x-ray scattering technique. Physical Review B, 2014. 90(24).

99. Wang, $C$., et al., Resonant soft $x$-ray reflectivity of organic thin films. Journal of Vacuum Science \& Technology A, 2007. 25(3): p. 575-586.

100. Yan, H.P., et al., Interfaces in organic devices studied with resonant soft $x$-ray reflectivity. Journal of Applied Physics, 2011. 110(10): p. 102220.

101. Swaraj, S., et al., The utility of resonant soft $x$-ray scattering and reflectivity for the nanoscale characterization of polymers. European Physical Journal-Special Topics, 2009. 167: p. 121-126.

102. Sunday, D.F., et al., Three-dimensional x-ray metrology for block copolymer lithography linespace patterns. Journal of Micro-Nanolithography Mems and Moems, 2013. 12(3).

103. Jones, R.L., et al., Small angle $x$-ray scattering for sub-100 $\mathrm{nm}$ pattern characterization. Applied Physics Letters, 2003. 83(19): p. 4059-4061.

104. Ingham, B., et al., Solving the mystery of the internal structure of casein micelles. Soft Matter, 2015. 11(14): p. 2723-2725.

105. Mata, J.P., P. Udabage, and E.P. Gilbert, Structure of casein micelles in milk protein concentrate powders via small angle X-ray scattering. Soft Matter, 2011. 7(8): p. 3837-3843.

106. Guo, C., et al., Signatures of Multiphase Formation in the Active Layer of Organic Solar Cells from Resonant Soft X-ray Scattering. Acs Macro Letters, 2013. 2(3): p. 185-189. 